Acta Crystallographica Section B

Structural

Science

ISSN 0108-7681

Iain D. H. Oswald, ${ }^{a}$ David R.

Allan, ${ }^{a}$ W. D. Samuel

Motherwell ${ }^{b}$ and Simon

Parsons $^{\text {a* }}$

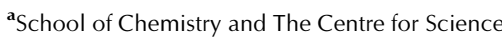
at Extreme Conditions, The University of Edinburgh, King's Buildings, West Mains Road, Edinburgh EH9 3JJ, Scotland, and ${ }^{\mathbf{b}}$ Cambridge Crystallographic Data Centre, 12 Union Road, Cambridge CB2 1EZ, England

Correspondence e-mail: s.parsons@ed.ac.uk

\title{
Structures of the monofluoro- and monochloro- phenols at low temperature and high pressure
}

2-Fluorophenol, 3-fluorophenol and 3-chlorophenol were recrystallized from frozen solids at 260, 263 and $283 \mathrm{~K}$. All compounds were also crystallized by the application of high pressure (0.36, 0.12 and $0.10 \mathrm{GPa})$. While 3-fluorophenol and 3 -chlorophenol yielded the same phases under both conditions, different polymorphs were obtained for 2-fluorophenol. 4-Chlorophenol was crystallized both from the melt and from benzene to yield two different ambient-pressure polymorphs; crystallization from the melt at $0.02 \mathrm{GPa}$ yielded the same phase as from benzene at ambient pressure. 3-Fluorophenol is unusual in forming a hydrogen-bonded chain along a $2_{1}$ screw axis. Such behaviour is usually only observed for small alcohols, but here it appears to be stabilized by intermolecular $\mathrm{C}-\mathrm{H} \cdots \mathrm{F}$ hydrogen-bond formation. 3-Chlorophenol is a more typical large alcohol and emulates a fourfold screw axis with two independent molecules positioned about a $2_{1}$ axis, although there are significant distortions from this ideal geometry. The two phases of 4-chlorophenol consist of chains or rings connected by $\mathrm{C}-\mathrm{Cl} \cdots \mathrm{H}$ interactions. The lowtemperature and high-pressure polymorphs of 2-fluorophenol consist of chains of molecules connected through $\mathrm{OH} \cdots \mathrm{OH}$ hydrogen bonds; while inter-chain $\mathrm{C}-\mathrm{H} \cdots \mathrm{F}$ interactions are significant at high pressure, there are none in the lowtemperature form.

\section{Introduction}

Packing in alcohols has been studied by Brock \& Duncan (1994) and subsequently by Taylor \& Macrae (2001). Both studies showed that the size of the $R$-group attached to the alcohol functionality is a major factor in the packing behaviour of the molecules. We have recently investigated (Oswald et al., 2005) the effect of high pressure on the crystal structures of phenol (Allan et al., 2002), 2-chlorophenol and 4-fluorophenol, which all exhibit polymorphic behaviour on application of pressure. At ambient pressure 2-chlorophenol and 4fluorophenol crystallize in high-symmetry space groups with the molecules disposed about $3_{2}$ and $\overline{3}$ symmetry operators; phenol packs via a pseudo-threefold axis in $P 2_{1}$. All three systems crystallize under pressure in low-symmetry space groups with the molecules disposed about $2_{1}$ screw axes. Fig. 1 illustrates the change in structure between ambient and high pressure for 2-chlorophenol. At ambient pressure the molecules adopt an arrangement in which the halophenyl group behaves as a bulky substituent. At high pressure, both compounds undergo a phase transition to a packing motif characteristic of a small alcohol. This paper describes the crystal structure determination of the remaining chloro- and
Received 23 August 2004

Accepted 23 November 2004
(C) 2005 International Union of Crystallography Printed in Great Britain - all rights reserved 
fluorophenols at low temperature and high pressure to investigate whether the packing behaviour of these phenols can be altered with the application of pressures $<1 \mathrm{GPa}$.

\section{Experimental}

All samples were obtained from Sigma-Aldrich and used as received.

\subsection{Low-temperature crystal growth}

2-Fluorophenol (m.p. 289 K), 3-fluorophenol (m.p. 287 K) and 3-chlorophenol (m.p. $306 \mathrm{~K}$ ) were each drawn into a capillary, and polycrystalline masses obtained by freezing at 260,263 and $283 \mathrm{~K}$, respectively. The samples were then recrystallized using the laser-assisted procedure of Boese \& Nussbaumer (1994). All capillaries (o.d. 0.32-0.52 mm) were hand-drawn from $4 \mathrm{~mm}$ o.d. Pyrex ${ }^{\circledR}$ glass tube. Phase I of 4chlorophenol (m.p. $316 \mathrm{~K}$ ) was obtained by melting a sample in a vial and leaving it to recrystallize at room temperature. Small, colourless crystals appeared on the side of the vial. Colourless crystals of phase II of 4-chlorophenol were obtained by holding a saturated benzene solution at $277 \mathrm{~K}$.

\subsection{Crystal structure determination at low temperature}

$\mathrm{X}$-ray diffraction intensities were collected with Mo $K \alpha$ radiation on a Bruker Smart Apex CCD diffractometer equipped with an Oxford Cryosystems Cryostream-Plus variable-temperature device (Cosier \& Glazer, 1986) and an OHCD laser-assisted crystallization device. Absorption corrections were carried out using the multiscan procedure $S A D A B S$ (Sheldrick, 2004, based on the procedure described by Blessing, 1995). All the structures were solved by direct methods (SIR92; Altomare et al., 1993) and refined by fullmatrix least-squares against $F^{2}$ using all data (CRYSTALS; Betteridge et al., 2003). $\mathrm{H}$ atoms were attached to $\mathrm{C}$ atoms in calculated positions and allowed to ride on their parent atoms. $\mathrm{H}$ atoms involved in hydrogen bonding were located in difference maps and refined with distance restraints. All non$\mathrm{H}$ atoms were modelled with anisotropic displacement parameters.

\subsection{High pressure: general procedures}

Pressure was applied to the samples using a Merrill-Bassett diamond anvil cell (DAC; Merrill \& Bassett, 1974) equipped with $600 \mu \mathrm{m}$ culets, a tungsten gasket with a $300 \mu \mathrm{m}$ hole, beryllium backing disks and a chip of ruby for pressure measurement. Pressures were measured by the ruby-fluorescence method by excitation with a $632.817 \mathrm{~nm}$ line from a HeNe laser using a Jobin-Yvon LabRam 300 Raman spectrometer.

\subsection{High-pressure crystal growth}

The samples were loaded as liquids into the cell. In the case of 4-chloro- and 4-fluorophenol, both the sample and the cell were heated with a hot-air gun before loading to prevent crystallization at ambient temperature. In each case, pressure was applied until a polycrystalline mass was produced; the temperature of the cell was increased using a hot-air gun until a single crystallite remained. Slow cooling to ambient temperature yielded a single crystal that filled the entire gasket hole. Crystallization was monitored visually using a polarizing microscope. The crystallization pressures for each sample were as follows: 3-chlorophenol, 0.10 GPa; 3-fluorophenol, 0.12 GPa; 4-chlorophenol, 0.02 GPa; 2-fluorophenol, $0.36 \mathrm{GPa}$.

\subsection{Crystal structure determinations at high pressure}

Data were collected on a Bruker SMART APEX diffractometer with Mo $K \alpha$ radiation. The collection and processing procedures followed were those described by Dawson et al. (2004).

Shading by the body of the DAC leads to low data completeness for crystals belonging to low-symmetry crystal systems. In all cases, except 2-fluorophenol, datasets were

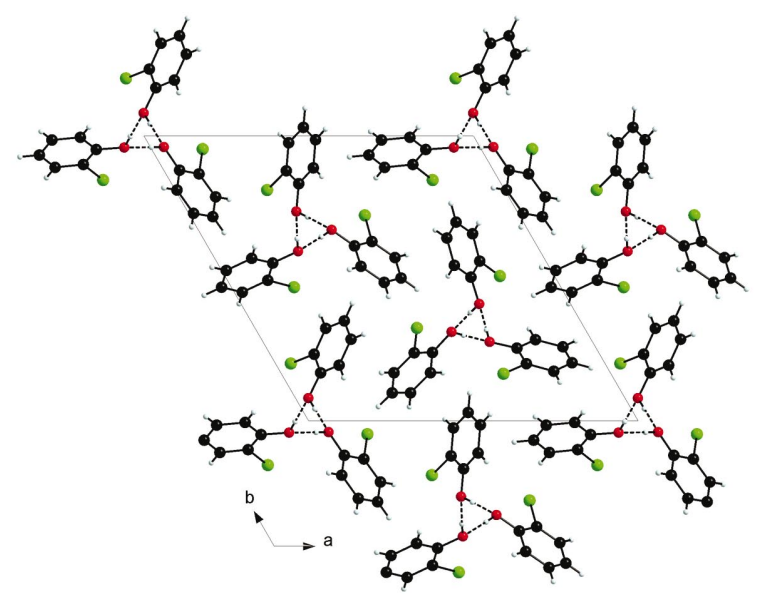

(a)

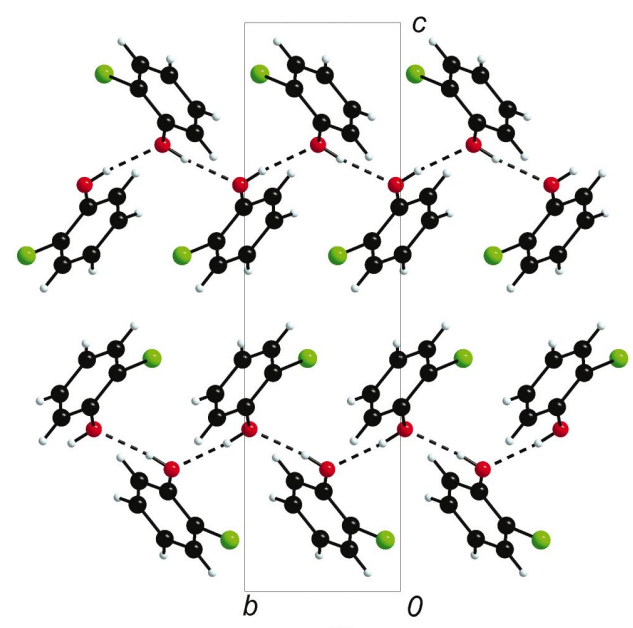

(b)

Figure 1

(a) Crystal structures of 2-chlorophenol at ambient pressure and $100 \mathrm{~K}$. Helices are formed about $3_{2}$ axes by $\mathrm{OH} \cdots \mathrm{OH}$ hydrogen-bond formation. (b) The crystal structure of 2-chlorophenol at $0.12 \mathrm{GPa}$. The application of pressure changes the behaviour of the chlorophenyl substituent so that chains are formed where molecules are related by a $2_{1}$ screw axis. Colour scheme: $\mathrm{C}$ black, $\mathrm{Cl}$ green, $\mathrm{H}$ light turquoise, $\mathrm{O}$ red. 
collected with the cell mounted in two different orientations in order to improve completeness. The diffraction patterns were indexed with the program GEMINI (Sparks, 2000). Data integration (to $2 \theta=45^{\circ}$ ) was performed using SAINT (Bruker-Nonius, 2003) with dynamic masking to account for the shading from the DAC steel body (ECLIPSE; Parsons, 2004a). The program SHADE (Parsons, 2004b) was also used to take account of absorption effects of the diamonds and beryllium; further systematic errors were treated using $S A D A B S$ before merging in SORTAV (Blessing, 1997).

The phases obtained for 3-fluorophenol, 3-chlorophenol and 4-chlorophenol corresponded to compressed forms of known ambient-pressure phases. Refinement against the highpressure data therefore used the ambient-pressure coordinates as a starting model.

The structures were refined by full-matrix least-squares against $F^{2}$ (CRYSTALS) using all data. Free refinement of the positional parameters of the non- $\mathrm{H}$ atoms yielded carboncarbon bond lengths varying from 1.34 to $1.40 \AA$. The phenyl rings were therefore constrained to be rigid hexagons. $\mathrm{H}$ atoms were attached to $\mathrm{C}$ atoms in calculated positions. The hydroxyl $\mathrm{H}$ atom, which is involved in hydrogen bonding, was geometrically placed except for 4-chlorophenol where the hydroxyl hydrogen was identified from the difference map and refined with distance and angular restraints. All oxygen and halogen atoms were modelled with anisotropic displacement parameters. The refinement of the crystal structure of 3fluorophenol was subject to distance and angle restraints. 2Fluorophenol, 3-chlorophenol and 4-chlorophenol were refined so that chemically similar bond distances and angles were subject to similarity restraints.

\subsection{2-Fluorophenol}

Several attempts to grow a single crystal of 2-fluorophenol at high pressure resulted in the crystal fracturing after cooling to ambient temperature. Although the diffraction patterns obtained from these samples were characterized by broad, split reflections, they could, nevertheless, be indexed on an orthorhombic unit cell with the dimensions: $a=5.8952$ (17), $b=10.9466$ (19), $c=16.459$ (4) $\AA$. This is different to the cell obtained at $150 \mathrm{~K}$ (see Table 1 ). A solution was obtained using DASH (David et al., 2001; see below), but after refinement the residual remained in the region of $R_{1}=0.17$. The refined structure, which contains two molecules in the asymmetric unit in the space group $P 2_{1} 2_{1} 2_{1}$, was characterized by high displacement parameters $\left(0.2-0.3 \AA^{2}\right)$ on the $\mathrm{F}$ atoms, while the data, although strong at low angle, had no significant intensity above about $2 \theta=35^{\circ}$. These observations imply that at $0.36 \mathrm{GPa}$ and room temperature 2-fluorophenol forms a disordered phase. Difference maps failed to provide any clue as to how the structure might be better modelled, probably because of the relatively low completeness or poor reflection peak shapes. Since we are unable to improve modelling of the data, no further details on this phase are reported here.

A new crystal was grown as above and then maintained at high temperature during data collection with the variable- temperature device set at $403 \mathrm{~K}$. This is a nominal temperature, as there was presumably a significant temperature gradient across the cell as a whole, although across the sample itself the variation in temperature would have been small. The high-temperature dataset indexed on a slightly smaller orthorhombic unit cell with the following dimensions: $a=$ 5.7168 (7), $b=9.9997$ (19), $c=17.868$ (2) A. Both the $b$ and $c$ axes show a large change in length compared with the ambient temperature/0.36 GPa cell given above $(\Delta b=+0.95 \AA ; \Delta c=$ $-1.41 \AA)$. This cell is also different from that obtained at low temperature (Table 1 ).

Conventional direct methods applied to the $403 \mathrm{~K}$ data set failed to yield a recognisable solution. This is a recurrent problem in high-pressure crystallography and is the result of low data-completeness. The problem can be overcome by using global optimization methods, originally devised for structure solution from powders. The crystal structure of 2fluorophenol at high pressure was therefore solved using the simulated annealing procedure in the program DASH (David et al., 2001). The refinement of the structure followed the procedures outlined in $\$ 2.5$.

\subsection{Recovery of 4-chlorophenol grown at high pressure}

The high melting point of 4-chlorophenol allowed the crystals of phase II formed at high pressure to be recovered without the sample melting. On release of the pressure the sample remained as a single crystal with a slight reduction in size due to melting around the edges. Diffraction data were collected at ambient pressure and $293 \mathrm{~K}$, which showed the crystal to be 4-chlorophenol Phase I (see Table 1).

\subsection{Software and other general procedures}

A consistent numbering scheme was used for all the structures described here and this is shown in (I). Where there is

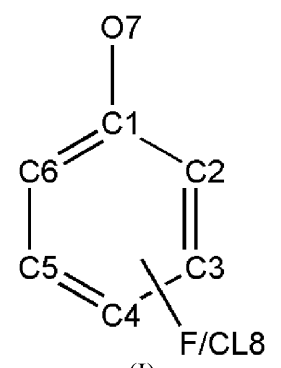

(I)

more than one molecule in the asymmetric unit the labels are augmented with the numbers 1,2 etc. A full listing of crystal, data collection and refinement parameters is given in Table 1, and a set of hydrogen-bonding parameters is given in Table 2 . The structures were visualized using SHELXTL (Sheldrick, 2001), MERCURY (Bruno et al., 2002) or CAMERON (Watkin et al., 1993); the figures were produced using DIAMOND (Crystal Impact, 2004). Other analyses utilized the p.c. version of PLATON (Spek, 2002; Farrugia, 1999). Searches of the Cambridge Structural Database (Allen, 2002; Allen \& Motherwell, 2002) were carried out with the program CONQUEST, utilizing Version 5.25 of the database. Calcu- 
Table 1

Experimental table.

Weighting scheme: $p=P(6) * \max \left(F_{o}^{2}, 0\right)+(1-P(6)) F_{c}^{2}$. Method $=$ SHELXL97 (Sheldrick, 1997).

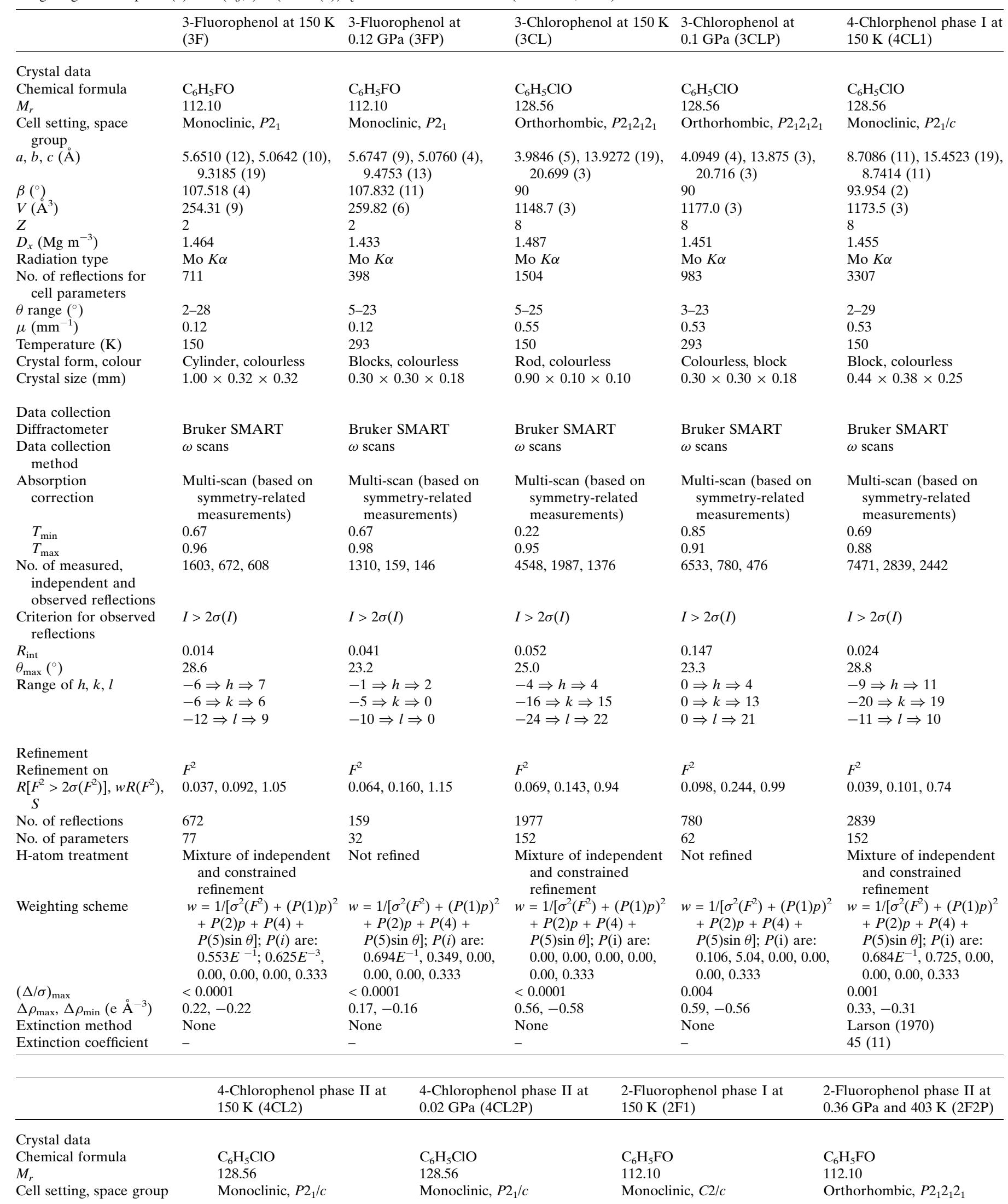


Table 1 (continued)

\begin{tabular}{|c|c|c|c|c|}
\hline & $\begin{array}{l}\text { 4-Chlorophenol phase II at } \\
150 \mathrm{~K} \text { (4CL2) }\end{array}$ & $\begin{array}{l}\text { 4-Chlorophenol phase II at } \\
0.02 \mathrm{GPa}(4 \mathrm{CL} 2 \mathrm{P})\end{array}$ & $\begin{array}{l}\text { 2-Fluorophenol phase I at } \\
150 \mathrm{~K}(2 \mathrm{~F} 1)\end{array}$ & $\begin{array}{l}\text { 2-Fluorophenol phase II at } \\
0.36 \mathrm{GPa} \text { and } 403 \mathrm{~K}(2 \mathrm{~F} 2 \mathrm{P})\end{array}$ \\
\hline$a, b, c(\AA)$ & $\begin{array}{l}3.9724(5), 12.7328(17) \\
23.155(3)\end{array}$ & $\begin{array}{l}4.1096(4), 12.7665(10), \\
23.181(3)\end{array}$ & $\begin{array}{l}17.1336(10), 8.2766(5), \\
11.4975(7)\end{array}$ & $\begin{array}{l}5.7168(7), 9.9997(19) \\
17.868(2)\end{array}$ \\
\hline$\beta\left(^{\circ}\right)$ & $94.126(2)$ & $94.201(14)$ & $100.234(2)$ & 90 \\
\hline$V\left(\AA^{6}\right)$ & $1168.2(3)$ & $1212.9(2)$ & $1604.50(17)$ & $1021.4(3)$ \\
\hline$Z$ & 8 & 8 & 12 & 8 \\
\hline$D_{x}\left(\mathrm{Mg} \mathrm{m}^{-3}\right)$ & 1.462 & 1.408 & 1.392 & 1.458 \\
\hline Radiation type & Mo $K \alpha$ & Mo $K \alpha$ & Mo $K \alpha$ & Mo $K \alpha$ \\
\hline $\begin{array}{l}\text { No. of reflections for cell } \\
\text { parameters }\end{array}$ & 2464 & 1170 & 2044 & 397 \\
\hline$\theta$ range $\left({ }^{\circ}\right)$ & $3-28$ & $3-19$ & $2-26$ & $3-20$ \\
\hline$\mu\left(\mathrm{mm}^{-1}\right)$ & 0.54 & 0.52 & 0.12 & 0.12 \\
\hline Temperature (K) & 150 & 293 & 150 & 403 \\
\hline Crystal form, colour & Plate, colourless & Block, colourless & Cylinder, colourless & Plate, colourless \\
\hline Crystal size (mm) & $0.70 \times 0.50 \times 0.36$ & $0.30 \times 0.30 \times 0.18$ & $1.00 \times 0.38 \times 0.38$ & $0.30 \times 0.30 \times 0.18$ \\
\hline \multicolumn{5}{|l|}{ Data collection } \\
\hline Diffractometer & Bruker SMART & Bruker SMART & Bruker SMART & Bruker SMART \\
\hline Data collection method & $\omega$ scans & $\omega$ scans & $\omega$ scans & $\omega$ scans \\
\hline Absorption correction & $\begin{array}{l}\text { Multi-scan (based on } \\
\text { symmetry-related } \\
\text { measurements) }\end{array}$ & $\begin{array}{l}\text { Multi-scan (based on } \\
\text { symmetry-related } \\
\text { measurements) }\end{array}$ & $\begin{array}{l}\text { Multi-scan (based on } \\
\text { symmetry-related } \\
\text { measurements) }\end{array}$ & $\begin{array}{l}\text { Multi-scan (based on } \\
\text { symmetry-related } \\
\text { measurements) }\end{array}$ \\
\hline$T_{\min }$ & 0.49 & 0.68 & 0.575 & 0.96 \\
\hline$T_{\max }$ & 0.82 & 0.91 & 1.000 & 0.98 \\
\hline $\begin{array}{l}\text { No. of measured, indepen- } \\
\text { dent and observed reflec- } \\
\text { tions }\end{array}$ & $7449,2843,2219$ & $7359,711,397$ & $7505,1957,1302$ & $3070,469,261$ \\
\hline $\begin{array}{l}\text { Criterion for observed } \\
\text { reflections }\end{array}$ & $I>2 \sigma(I)$ & $I>2 \sigma(I)$ & $I>2 \sigma(I)$ & $I>2 \sigma(I)$ \\
\hline$R_{\text {int }}$ & 0.016 & 0.063 & 0.021 & 0.099 \\
\hline$\theta_{\max }\left({ }^{\circ}\right)$ & 28.9 & 23.2 & 28.7 & 23.3 \\
\hline Range of $h, k, l$ & $-4 \Rightarrow h \Rightarrow 5$ & $-4 \Rightarrow h \Rightarrow 4$ & $-22 \Rightarrow h \Rightarrow 22$ & $0 \Rightarrow h \Rightarrow 6$ \\
\hline & $-14 \Rightarrow k \Rightarrow 17$ & $0 \Rightarrow k \Rightarrow 14$ & $-10 \Rightarrow k \Rightarrow 11$ & $0 \Rightarrow k \Rightarrow 5$ \\
\hline & $-31 \Rightarrow l \Rightarrow 29$ & $0 \Rightarrow l \Rightarrow 10$ & $-15 \Rightarrow l \Rightarrow 15$ & $0 \Rightarrow l \Rightarrow 19$ \\
\hline \multicolumn{5}{|l|}{ Refinement } \\
\hline Refinement on & $F^{2}$ & $F^{2}$ & $F^{2}$ & $F^{2}$ \\
\hline$R\left[F^{2}>2 \sigma\left(F^{2}\right)\right], w R\left(F^{2}\right), S$ & $0.043,0.110,0.75$ & $0.055,0.160,1.03$ & $0.056,0.111,1.07$ & $0.083,0.251,0.94$ \\
\hline No. of reflections & 2843 & 710 & 1956 & 461 \\
\hline No. of parameters & 151 & 61 & 125 & 62 \\
\hline H-atom treatment & $\begin{array}{l}\text { Mixture of independent and } \\
\text { constrained refinement }\end{array}$ & Not refined & $\begin{array}{l}\text { Mixture of independent and } \\
\text { constrained refinement }\end{array}$ & Not refined \\
\hline Weighting scheme & $\begin{array}{l}w=1 /\left[\sigma^{2}\left(F^{2}\right)+(P(1) p)^{2}+\right. \\
\quad P(2) p+P(4)+P(5) \sin \theta] \\
\quad P(i) \text { are: } 0.700 E^{-1}, 0.890 \\
\quad 0.00,0.00,0.00,0.333\end{array}$ & $\begin{array}{l}w=1 /\left[\sigma^{2}\left(F^{2}\right)+(P(1) p)^{2}+\right. \\
\quad P(2) p+P(4)+P(5) \sin \theta] \\
P(\mathrm{i}) \text { are: } 0.625 E^{-1}, 1.91 \\
\quad 0.00,0.00,0.00,0.333\end{array}$ & $\begin{array}{l}w=1 /\left[\sigma^{2}\left(F^{2}\right)+(P(1) p)^{2}+\right. \\
\quad P(2) p+P(4)+P(5) \sin \theta] \\
\quad P(i) \text { are: } 0.172 E^{-1}, 1.34 \\
\quad 0.00,0.00,0.00,0.333\end{array}$ & $\begin{array}{l}w=1 /\left[\sigma^{2}\left(F^{2}\right)+(P(1) p)^{2}+\right. \\
\quad P(2) p+P(4)+P(5) \sin \theta] \\
\quad P(i) \text { are: } 0.173,0.00,0.00 \\
\quad 0.00,0.00,0.333\end{array}$ \\
\hline$(\Delta / \sigma)_{\max }$ & 0.001 & 0.007 & $<0.0001$ & 0.001 \\
\hline 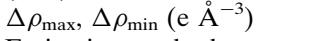 & $0.44,-0.44$ & $0.21,-0.20$ & $0.32,-0.40$ & $0.27,-0.20$ \\
\hline Extinction method & None & None & Larson (1970) & None \\
\hline Extinction coefficient & - & - & $29(9)$ & - \\
\hline
\end{tabular}

lations involving projected vectors followed the methods of Sands (1995). Crystallographic information files for all structures reported here are available as supplementary material. ${ }^{\mathbf{1}}$

\section{Results}

\subsection{3-Fluorophenol}

3 -Fluorophenol crystallizes at $263 \mathrm{~K}$ in the space group $P 2_{1}$ with one molecule in the asymmetric unit. Diffraction data were collected at $150 \mathrm{~K}$. The molecules interact via

\footnotetext{
${ }^{1}$ Supplementary data for this paper are available from the IUCr electronic archives (Reference: BS5012). Services for accessing these data are described at the back of the journal.
}

... OH...OH... hydrogen bonds to form chains disposed about the crystallographic $2_{1}$ screw axes, conforming to a $C(2)$ graph set (Fig. 2a). This packing motif is more commonly associated with small alcohols and was quite unexpected. It appears that stabilization of this motif occurs through the formation of $\mathrm{H} 6 \cdots \mathrm{F} 8$ interactions $(2.61 \AA)$ between the chains (Fig. 2b). Taken on their own these $\mathrm{C}-\mathrm{H} \cdots \mathrm{F}$ interactions form chains which run along the $\langle 110\rangle$ directions. The $\mathrm{OH}$... O hydrogen bond present in this system is slightly longer [O7 ...O $7^{\prime} 2.819$ (1) $\AA$ ] than those present in the other systems described in this paper. In projection onto (010) each chain is surrounded by six others.

The same phase is obtained on crystallization at $0.12 \mathrm{GPa}$. Neither the hydrogen bond nor the stabilizing $\mathrm{C}-\mathrm{H} \cdots \mathrm{F}$ 
Table 2

Hydrogen-bonding parameters ( $)$.

The hydrogen-bonding distances are from donor to acceptor due to the imprecise determination of $\mathrm{H}$-atom positions. $\mathrm{H} \cdots \mathrm{Cl}$ and $\mathrm{H} \cdots \mathrm{F}$ distances are given before and after the normalization of $\mathrm{C}-\mathrm{H}$ distances to typical neutron values $(1.083 \AA$ ), the latter being given in square brackets. The high-pressure crystal structure of 2-fluorophenol was determined at $403 \mathrm{~K}$.

\begin{tabular}{|c|c|c|c|c|}
\hline \multirow[b]{2}{*}{ Compound } & \multirow[b]{2}{*}{ Donor } & \multirow[b]{2}{*}{ Acceptor } & \multicolumn{2}{|l|}{$D \cdots A$ distance $(\AA)$} \\
\hline & & & $\begin{array}{l}\text { Low-temperature } \\
(150 \mathrm{~K})\end{array}$ & High pressure \\
\hline \multirow[t]{2}{*}{$3-\mathrm{F}$} & O7 & $\mathrm{O} 7^{\mathrm{i}}$ & 2.819 (1) & $2.843(8)$ \\
\hline & H6 & $\mathrm{F}^{\mathrm{ii}}$ & $2.61[2.48]$ & 2.62 [2.49] \\
\hline \multirow[t]{2}{*}{ 3-Cl } & O71 & $\mathrm{O} 72^{\mathrm{iii}}$ & $2.734(7)$ & 2.693 (4) \\
\hline & $\mathrm{O} 72$ & O71 & $2.700(6)$ & $2.753(4)$ \\
\hline \multirow[t]{4}{*}{ 4-Cl phase I } & O71 & $\mathrm{O} 72^{\mathrm{iv}}$ & $2.767(2)$ & - \\
\hline & $\mathrm{O} 72$ & O71 & $2.779(2)$ & - \\
\hline & H62 & $\mathrm{Cl} 81^{\mathrm{v}}$ & $2.93[2.82]$ & - \\
\hline & $\mathrm{H} 21$ & $\mathrm{Cl} 82^{\mathrm{vi}}$ & $2.93[2.82]$ & - \\
\hline \multirow{3}{*}{ 4-Cl phase II } & O71 & O72 & $2.762(2)$ & $2.819(5)$ \\
\hline & $\mathrm{O} 72$ & O71 & $2.779(2)$ & $2.749(5)$ \\
\hline & $\mathrm{H} 31$ & $\mathrm{Cl} 82^{\text {viii }}$ & $2.83[2.73]$ & $2.85[2.75]$ \\
\hline \multirow[t]{4}{*}{ 2-F phase I } & $\mathrm{O} 71$ & $\mathrm{O} 71^{\mathrm{ix}}$ & $2.774(3)$ & - \\
\hline & O71 & $\mathrm{O} 72$ & $2.707(2)$ & - \\
\hline & O71 & F81 & $2.690(2)$ & - \\
\hline & O71 & F81 $1^{\text {ix }}$ & $2.942(2)$ & - \\
\hline \multirow[t]{6}{*}{ 2-F phase II } & $\mathrm{O} 71$ & $\mathrm{O} 72^{\mathrm{x}}$ & - & $2.861(7)$ \\
\hline & $\mathrm{O} 72$ & O71 & & $3.097(8)$ \\
\hline & F81 & $\mathrm{H} 62^{\mathrm{xi}}$ & & $2.56[2.46]$ \\
\hline & F82 & $\mathrm{H} 61^{\mathrm{xii}}$ & & $2.56[2.49]$ \\
\hline & F82 & $\mathrm{H} 52^{\mathrm{xiii}}$ & & $2.63[2.53]$ \\
\hline & O71 & F82 & & $2.941(7)$ \\
\hline
\end{tabular}

Symmetry codes: (i) $-x, \frac{1}{2}+y,-z$; (ii) $1+x, 1+y, z$; (iii) $x-\frac{1}{2},-\frac{1}{2}-y,-2-z$; (iv) $x, \frac{3}{2}-y, \frac{1}{2}+z$; (v) $2-x, y-\frac{1}{2},-\frac{1}{2}-z$; (vi) $1+x, y, z$; (vii) $2-x, 1-y, 1-z$; (viii) $1-x, 2-y, 1-z$; (ix) $1-x,-y, 1-z$; (x) $\frac{1}{2}+x, \frac{1}{2}-y, 1-z$; (xi) $-\frac{1}{2}+x, \frac{1}{2}-y, 1-z$; (xii) $-1+x, y, z ;\left(\right.$ (xiii) $-x, \frac{1}{2}+y, \frac{3}{2}-z$.

interaction are significantly different to those in the low-

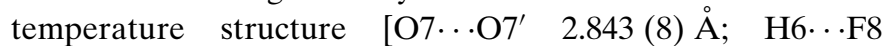
$2.62 \AA]$.

\subsection{3-Chlorophenol}

3-Chlorophenol is a liquid under ambient pressure with a melting point of $306 \mathrm{~K}$. At $283 \mathrm{~K}$ it crystallizes in space group $P 2{ }_{1} 2_{1} 2_{1}$ with two molecules in the asymmetric unit; diffraction data were collected at $150 \mathrm{~K}$. Primary bond distances and angles are normal, and are available as supplementary material. The molecules interact via $\cdots \mathrm{OH} \cdots \mathrm{OH} \cdots$ hydrogen bonds to form pseudo-fourfold helical chains (Fig. 3a). The two crystallographically independent molecules alternate along the chains. The angle between successive $\mathrm{C} 11-\mathrm{O} 71$ and $\mathrm{C} 12-\mathrm{O} 72$ vectors in the chain when projected onto the (100) plane is $89.75^{\circ}$; in a perfect fourfold helix this value would be $90^{\circ}$. While the departure from projected fourfold symmetry in the $\mathrm{OH} \cdots \mathrm{OH} \cdots$ interaction is slight, the orientations of the chloro groups do not conform to the pseudo-symmetry - the angle between $\mathrm{Cl}-\mathrm{C}$ vectors projected onto (100) is $17.74^{\circ}$. In addition, the molecules are not regularly spaced along the helix; the separations between the $\mathrm{O}$ atoms in the $a$ direction are 0.44 or $1.55 \AA$ (Fig. $3 b$ ).
The chains conform to a $C_{2}^{2}(4)$ graph set and are disposed about the $2_{1}$ axes parallel to the $a$-axis direction (Fig. $3 a$ ). The two crystallographically independent hydrogen bonds are

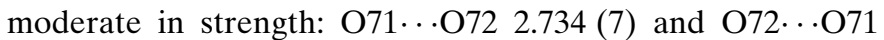
2.700 (6) $\AA$ (Table 2). The chains appear to be close-packed when viewed in projection onto (100) and, in contrast to the fluoro derivative, there are no contacts between the chains that fall within the sums of the van der Waals radii.

Crystallization at $0.1 \mathrm{GPa}$ results in the same structure as that at ambient pressure. The interactions between molecules are significantly different to those at ambient pressure

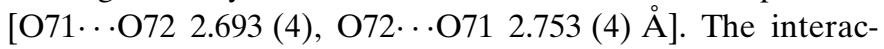
tion between $\mathrm{O} 71 \cdots \mathrm{O} 72$ appears to decrease in length at pressure while $\mathrm{O} 72 \cdots \mathrm{O} 71$ increases, although it is not possible to differentiate between the effects of pressure and temperature since the ambient pressure structure was determined at $150 \mathrm{~K}$, while the high-pressure determination was at room temperature.

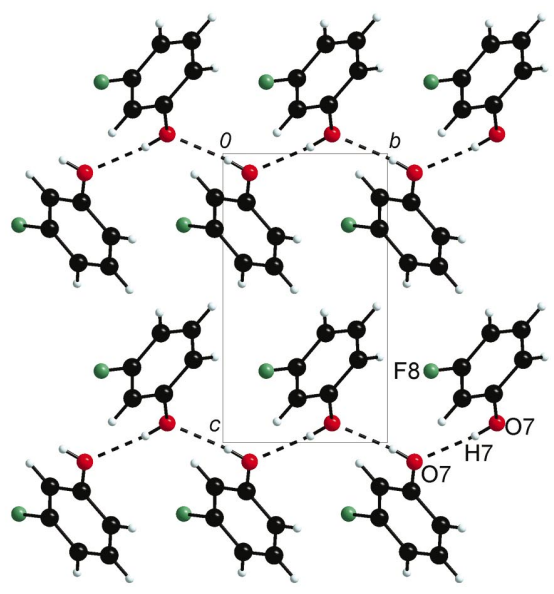

(a)

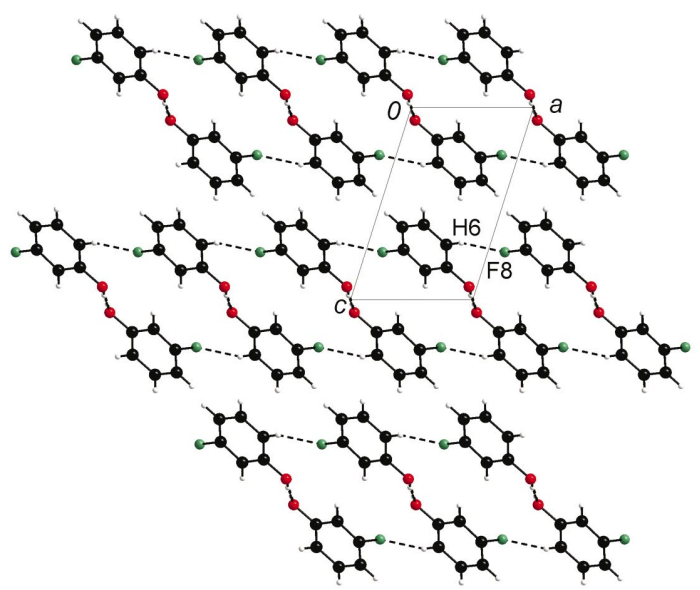

(b)

Figure 2

(a) Crystal structure of 3-fluorophenol at $150 \mathrm{~K}$ viewed down the $a$ axis. 3Fluorophenol crystallizes with one molecule in the asymmetric unit in the space group $P 2_{1}$, thereby forming a chain motif adopted by smaller alcohols. ( $b$ ) Close contacts formed between H6 ․ F8 of different chains (these contacts are omitted in Fig. $2 a$ for clarity). Colour scheme: C black, $\mathrm{F}$ sea green, $\mathrm{H}$ light turquoise, $\mathrm{O}$ red. 


\subsection{4-Chlorophenol phase I at $150 \mathrm{~K}$}

4-Chlorophenol is a solid at room temperature and was characterized by Perrin \& Michel $(1973 a, b)$. It crystallizes in two polymorphic forms and the structures of these have been redetermined at $150 \mathrm{~K}$ as part of this study.

Phase I crystallizes from the melt at ambient pressure in the space group $P 2_{1} / c$ with two molecules in the asymmetric unit; it is the more stable of the two phases. The two independent molecules alternate along the $c$ direction, forming $\mathrm{OH}$... OH. . hydrogen bonds resulting in a $C_{2}^{2}(4)$ graph set (Fig. 4a). The hydrogen bonds formed in this structure are of

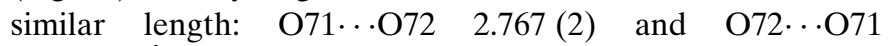
2.779 (2) A.

The graph-set descriptor is the same as that in 3-chlorophenol, but the chain is built by successive application of $c$ glide operations rather than a screw axis. Although the chain is helical, with a repeat at every fourth molecule, the pseudofourfold symmetry is even less ideal than in 3-chlorophenol
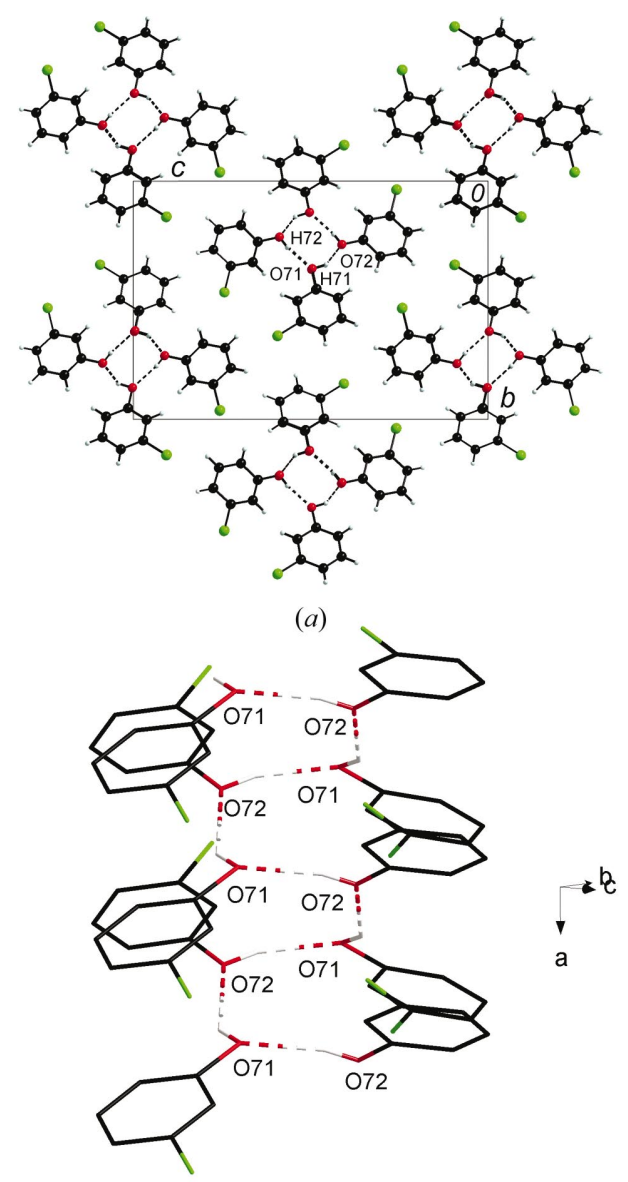

(b)

Figure 3

(a) Crystal structure of 3-chlorophenol at $150 \mathrm{~K}$. Distorted pseudofourfold helices are formed through $\mathrm{OH}$. . OH hydrogen bonds between the two molecules in the asymmetric unit. The structure at high pressure $(0.1 \mathrm{GPa})$ is similar to the low-temperature structure. (b) Although in projection on (100), the positions of the $\mathrm{C}-\mathrm{O}$ bonds resemble those in a fourfold helix and the positions of the molecules along the axis of the helix are irregular. Colour scheme as in Fig. 1.
(Fig. 4b). The angle between $\mathrm{O} 71-\mathrm{C} 11$ and $\mathrm{O} 72-\mathrm{C} 12$ when projected onto $(010)$ is $133.17^{\circ}$, which compares with $89.75^{\circ}$ in 3 -chlorophenol. Moreover, the spacing of molecules along the direction of the helix is irregular: the spacings of the $\mathrm{O}$ atoms in the $c$ direction are 1.98 and $2.39 \AA$.

The chains are linked by $\mathrm{H} 21 \cdots \mathrm{Cl} 82$ and $\mathrm{H} 62 \cdots \mathrm{Cl} 81$ interactions, both measuring $2.93 \AA$ (Fig. 4b). Taken on their own these $\mathrm{C}-\mathrm{Cl} \cdots \mathrm{H}$ interactions build spiral chains which are disposed about the $2_{1}$ axis parallel to b. Neighbouring chains interact with one another through a $\pi$-stacking interaction between pairs of symmetry-equivalent molecules containing $\mathrm{C} 11-\mathrm{Cl} 81$. The distance between the phenyl ring planes is $3.45 \AA$ with the centroids separated by $3.77 \AA$, which equates to a $1.74 \AA$ centroid displacement.

\subsection{4-Chlorophenol phase II at $150 \mathrm{~K}$ and $0.02 \mathrm{GPa}$}

The second phase of 4-chlorophenol is obtained by recrystallization from benzene; it is in the same space group as phase
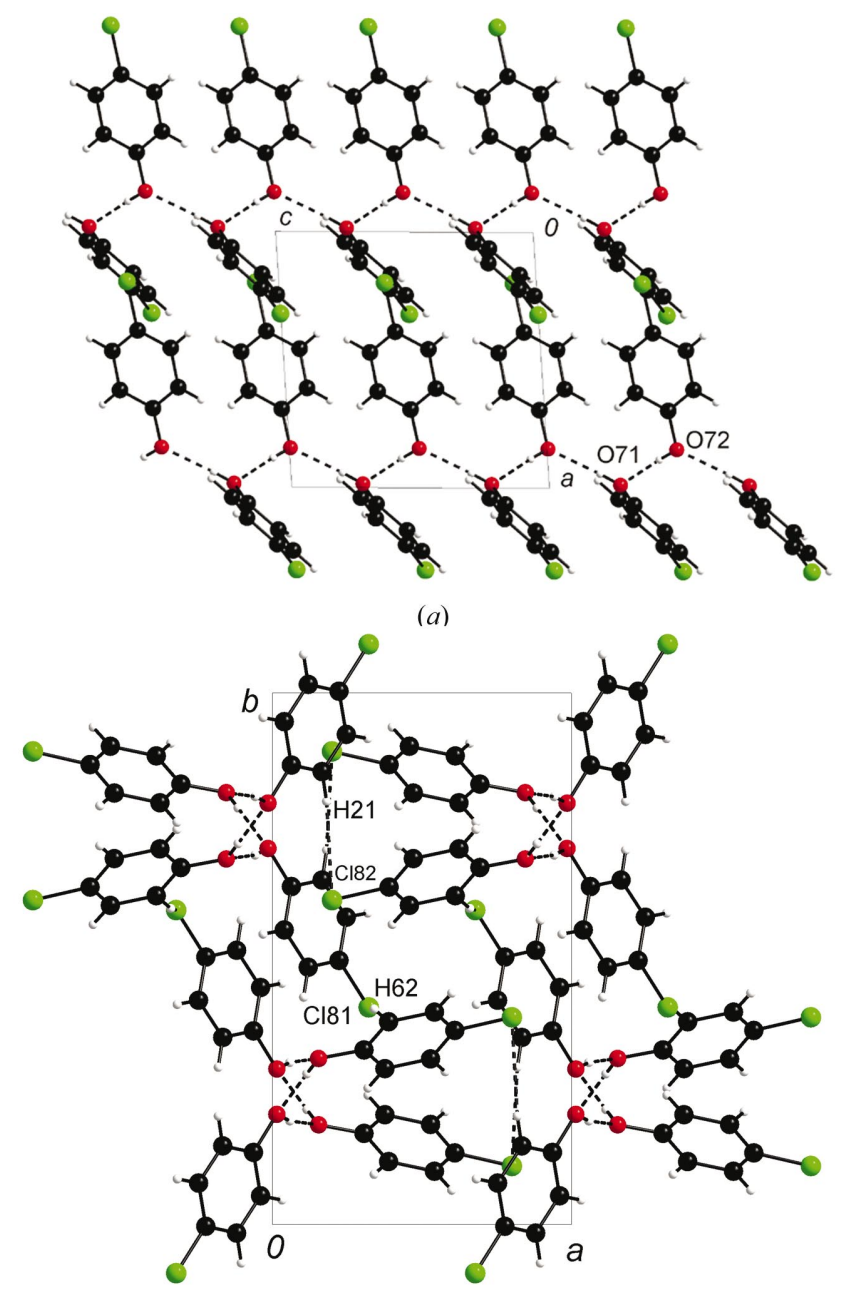

(b)

Figure 4

(a) Crystal structure of phase I of 4-chlorophenol at $150 \mathrm{~K}$ viewed along the $b$ axis, showing chains of molecules linked by $\mathrm{OH}$... OH hydrogen bonds. (b) View down [001], along the chains. The $\mathrm{Cl} \cdots \mathrm{H}$ interactions occur between $\mathrm{Cl} 82 \cdots \mathrm{H} 21$ and $\mathrm{Cl} 81 \cdots \mathrm{H} 62$; the latter lie parallel to the $b$ axis and run into the page. Colour scheme as in Fig. 1. 
I, $P 2_{1} / c$, with two molecules in the asymmetric unit. Phase II is metastable and transforms spontaneously to phase I if placed in contact with it (Perrin \& Michel, 1973b).

As in phase I, the molecules interact via $\cdots \mathrm{OH} \cdots \mathrm{OH} \cdots$ hydrogen bonds, but the interactions form an $R_{4}^{4}(8)$ graph set rather than hydrogen-bonded chains (Fig. 5). These rings stack along the $a$ direction. The hydrogen bonds are of a similar

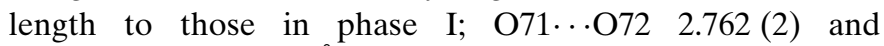
O72...O71 2.779 (2) $\AA$. Within the $R_{4}^{4}(8)$ rings a secondary $\mathrm{CH} \cdots \pi$ interaction is formed between $\mathrm{H} 21$ and the $\pi$-system

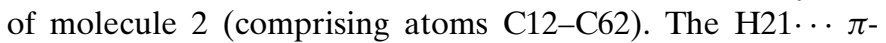
centroid is $3.95 \AA$, near to the limit for these interactions ( $4 \AA$ ) as defined by Malone et al. (1997), and it adopts a 'Type V' motif, as defined by the same authors.

There are close contacts between $\mathrm{Cl}$ and $\mathrm{H}$ atoms (Cl82 . .H31 $2.83 \AA$ ․ sum of van der Waals' $2.95 \AA)$ that join the $R_{4}^{4}(8)$ groups together into a ribbon. The ribbons lie along the [110] direction at $c=\frac{1}{2}$ and the [110] direction at $c=0,1$ etc.

Under ambient conditions crystallization of 4-chlorophenol from the melt yields phase I, but when crystallized from the melt under pressure $(0.02 \mathrm{GPa})$, phase II is formed. This pressure is very slight indeed by the standards of high-pressure crystallography and is barely measurable using the ruby fluorescence technique. The molecular arrangement is the same as the ambient pressure structure, although $\mathrm{O} 71 \cdots \mathrm{O} 72$ is significantly longer than at ambient pressure [2.819 (5) $\AA$ ], while $\mathrm{O} 72 \cdots \mathrm{O} 71$ is significantly shorter $[2.749(5) \AA]$. A similar effect was observed in 3-chlorophenol.

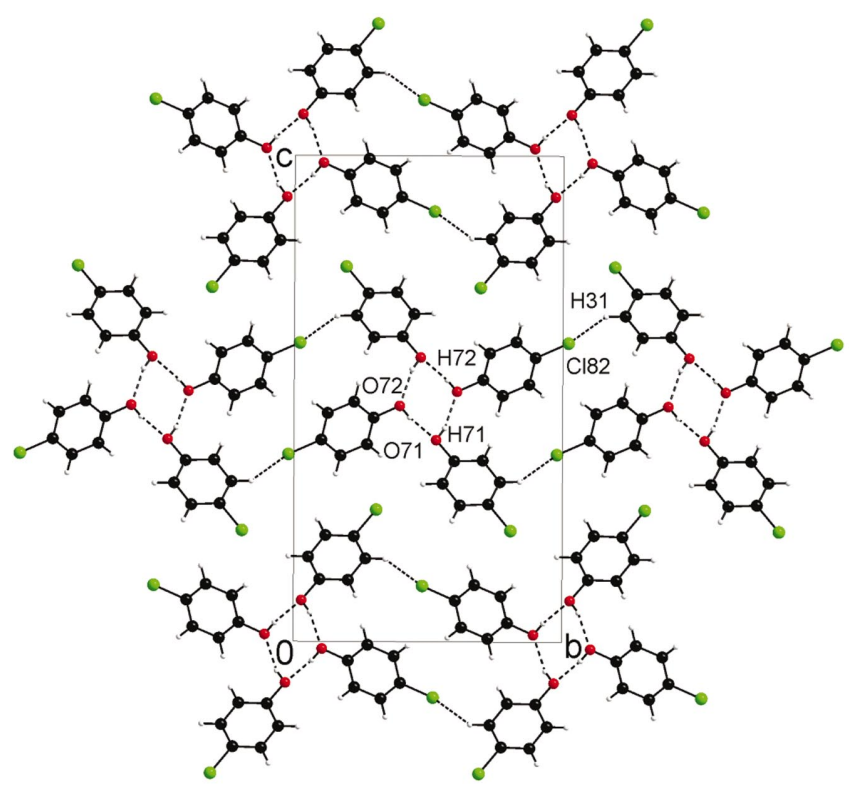

Figure 5

Crystal structure of phase II of 4-chlorophenol at $150 \mathrm{~K}$ viewed along the $a$ axis. The molecules crystallize in a ring motif that forms around an approximate fourfold axis. The discrete hydrogen-bonded groups are linked through an interaction between $\mathrm{Cl} 82 \cdots \mathrm{H} 31$. The structure at high pressure is similar to the low-temperature structure. Colour scheme as in Fig. 1.
The crystal of phase II grown at high pressure transformed to a single crystal of phase I when the pressure was released, but this transformation is not reversible, i.e. applying hydrostatic pressure to a crystal of phase I does not yield phase II. It is possible that the phase II-to-I transformation occurs by conversion of the $R_{4}^{4}(8)$ ring motifs, which are stacked by lattice repeats along the $a$ direction in phase $\mathrm{I}$, into $C_{4}^{4}(8)$ chains, developed by a $c$ glide, in phase II. Such a change in the intermolecular interactions would approximately double the length of the lattice repeat in the $a$ direction in going from phase I to II $(a=3.97$ and $c=8.74 \AA$ in phases I and II, respectively). In both phases $\mathrm{C}-\mathrm{Cl} \cdots \mathrm{H}$ interactions build chains which spiral along the $2_{1}$-axes along the $b$ directions. These similarities presumably promote the preservation of the single crystal through the phase transition.

\subsection{2-Fluorophenol phase I at $150 \mathrm{~K}$}

2-Fluorophenol crystallizes in space group $C 2 / c$ with oneand-a-half molecules in the asymmetric unit. We refer to this

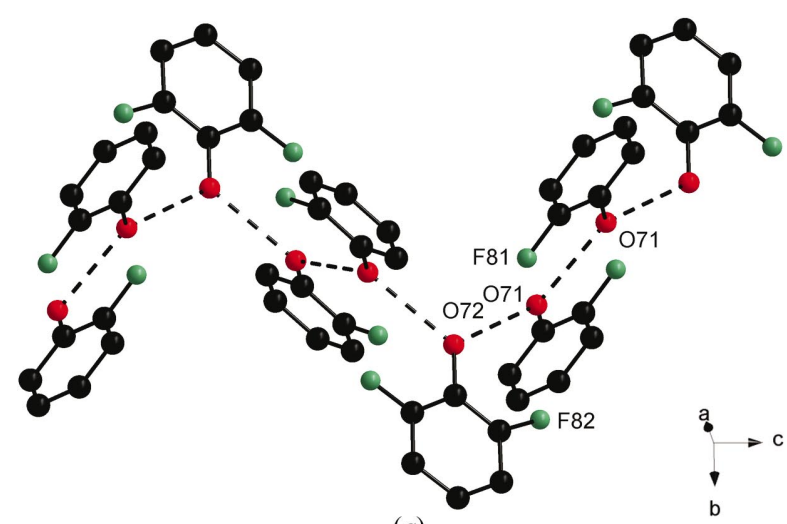

(a)
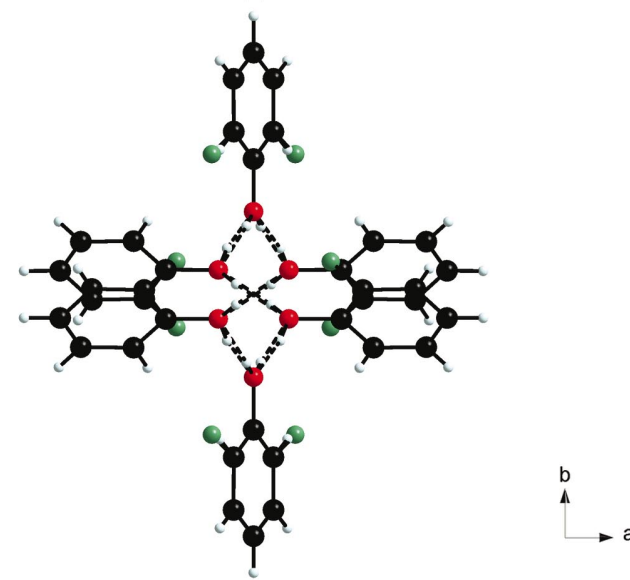

(b)

Figure 6

(a) Crystal structure of phase I of 2-fluorophenol at $150 \mathrm{~K}$ showing the disordered hydrogen-bonded chain. Molecule 1 forms a dimer with a symmetry-equivalent molecule. These dimers are linked through a hydrogen-bonding interaction to molecule $2 . \mathrm{H}$ atoms are omitted for clarity. F82 is disordered by the twofold axis which runs through O72C12. (b) View down the hydrogen-bonded chain. Colour scheme as in Fig. 2 . 
phase as phase I. One molecule (molecule 1, C11-F81) occupies a general position and is ordered. A second molecule (molecule 2, C12-F82) occupies a twofold axis with the axis running through atoms $\mathrm{O} 72 \cdots \mathrm{C} 12 \cdots \mathrm{C} 42$, and so the hydroxyl hydrogen and the $\mathrm{F}$ atoms are disordered.

The molecules interact via $\cdots \mathrm{OH} \cdots \mathrm{OH} \cdots$ hydrogen bonds forming chains; the direction of the hydrogen bonding in these chains must be disordered as a result of the disorder in molecule 2. A pair of molecules of type 1 are connected by a hydrogen bond formed across an inversion centre. These 'dimer' sub-units are then bridged by disordered molecules of type 2 (Fig. $6 a$ ). When projected along a the chains have a marked zigzag structure. When viewed in projection along $\mathbf{c}$ the chain of molecules somewhat resembles a helix, but the resemblance is an artefact of the projection: the displacement along $\mathbf{c}$ of one molecule to another is quite irregular, being $2.18 \AA$ across the inversion centre and $5.75 \AA$ across the twofold axis, while the angles made between successive OC vectors alternate between 180 and $89.83^{\circ}$ (Fig. 6b). The hydrogen-bond lengths are similar to those observed for other

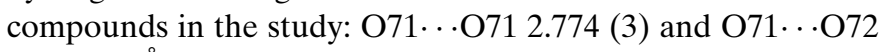
2.707 (2) $\AA$. There are $\pi$-stacking interactions between molecule 1 and a symmetry equivalent in the next chain along the $a$ axis. The phenyl rings lie parallel to one another $3.62 \AA$ apart with a centroid displacement in the plane of the ring of $1.48 \AA$.

\subsection{2-Fluorophenol phase II at $0.36 \mathrm{GPa}$ and $403 \mathrm{~K}$}

A crystal of 2-fluorophenol was grown at $0.36 \mathrm{GPa}$, but the crystal fractured a few hours after cooling to ambient temperature. Despite the poor X-ray diffraction data, the diffraction pattern could be indexed on an orthorhombic unit cell $(a=5.8952, b=10.9466, c=16.459 \AA)$; this is different to that determined at ambient pressure at $150 \mathrm{~K}$, indicating that a different phase had formed under high pressure. A tentative structural solution was obtained, but the refinement residuals were unacceptably high and it is likely that on cooling to room temperature the compound forms a disordered phase.

We found that the crystal obtained at high pressure was stable if the cell was held above ca $363 \mathrm{~K}$ and so a data collection was carried out in which the cell was held at $403 \mathrm{~K}$. This phase of 2-fluorophenol crystallizes in the space group $P 22_{1} 2_{1} 2_{1}$ with two molecules in the asymmetric unit. In one molecule the $\mathrm{C}-\mathrm{F}$ bond refined to an unrealistically short distance, which may indicate high librational disorder of the molecule. This is not unreasonable for a structure at $403 \mathrm{~K}$, although it is difficult to assess from the displacement parameters because of the low data completeness which resulted from shading by the pressure cell.

Oxygen and fluorine have similar X-ray scattering factors and so assignment of these sites was made on the basis of interatomic contacts. The assumption that $\mathrm{O}$ atoms are likely to make at least one hydrogen bond in which the distance between the non- $\mathrm{H}$ atoms is between 2.6 and $3.1 \AA$ A serves to identify O71 as an oxygen atom. The shortest contact (3.37 $\AA$ ) made by F81 is to C62 in a neighbouring ring; this distance is similar to those quoted by Thalladi et al. (1998) for C...F distances in $\mathrm{C}-\mathrm{H} \cdots \mathrm{F}$ hydrogen bonds, which therefore lends support to the assignment.

Atom assignments, $\mathrm{O} 72$ and F82, in the second of the two independent molecules were made by the refinement of two alternative models with part-weight hydroxyl $\mathrm{H}$ atoms placed in two alternative positions on each $\mathrm{O}$ atom. The $R 1$ factors for the model presented here and the alternative were 0.121 and 0.124 , respectively. The occupancies of alternative $\mathrm{H}$-atom positions refined to $0.85: 0.15$ (13); only the $\mathrm{H}$ atoms of major occupancy were retained. The alternative model contains $\mathrm{OH} \cdots \mathrm{F}$ interactions; organic fluorine is not expected to be competitive with hydroxyl oxygen as a hydrogen-bond acceptor.

The $\mathrm{OH} \cdots \mathrm{OH} \cdots$ chain that is formed (along the $a$ direction) conforms to a $C_{2}^{2}(4)$ graph set. The $\mathrm{O} 71 \cdots \mathrm{O} 72$ distances are 2.861 (7) and 3.097 (8) $\AA$. In common with other structures in this series, each chain is surrounded by six others and there are F82..H52 interactions formed between the chains $(2.63 \AA$, Fig. $7 a)$. The $\mathrm{O} \cdots \mathrm{O}$ distance of $3.097(8) \AA$ is the longest observed for an $\mathrm{OH} \cdots \mathrm{OH}$ interaction in this series and the length of the interaction may reflect the steric effect of F72 [O71 ‥F72 2.941 (7) $\AA$ ]. This arrangement is stabilized by

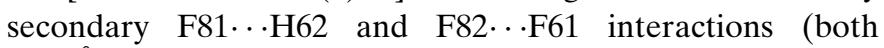
$2.56 \AA$ ) formed within the chains (Fig. $7 b$ ).

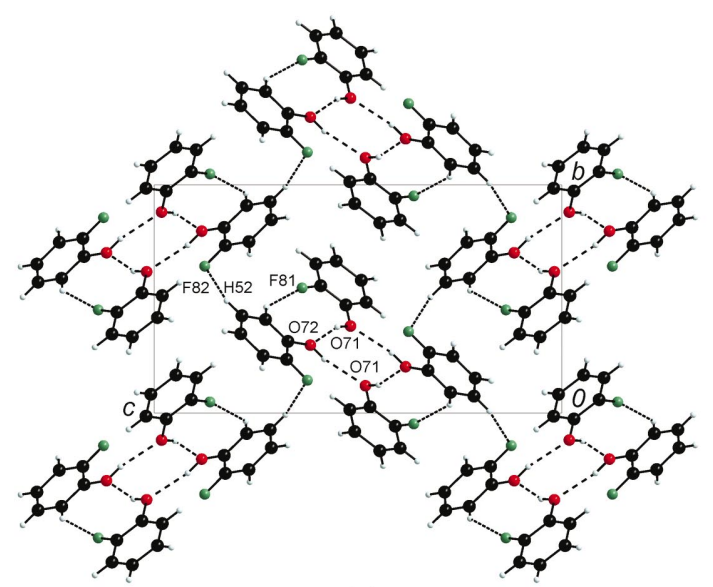

(a)

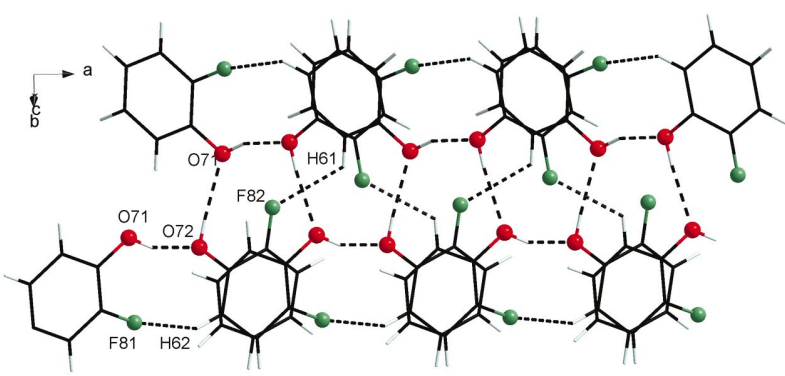

(b)

Figure 7

(a) Crystal structure of phase II of 2-fluorophenol at $0.36 \mathrm{GPa}$ viewed along a showing chains of molecules linked by $\mathrm{OH} \cdots \mathrm{OH}$ hydrogen bonds. The chains are linked by $\mathrm{C}-\mathrm{H} \cdots \mathrm{F}$ hydrogen bonds between $\mathrm{H} 52$ and F82. (b) The hydrogen-bonded chains viewed side-on. Colour scheme as in Fig. 2. 


\section{Discussion}

Compounds that are crystallized under ambient conditions and then subjected as single crystals to high pressure may be reduced to powder during a phase transition. Low-melting compounds are therefore useful for the study of pressureinduced polymorphism because high-pressure phases can be accessed directly by in situ crystallization in a high-pressure cell. A particularly striking example of the simplification in phase behaviour that can be gained by in situ crystallization is that of $S_{8}$ : the solid-region temperature/pressure phase diagram contains no less than 12 different phases when studied by compression of a solid sample, but there are only three when the liquid is allowed to crystallize from the melt (Mezouar, 2001). The series of molecules reported herein was chosen for its well defined hydrogen-bonding characteristics and the rigidity of the molecules.

We have described the structures of 2-chlorophenol and 4fluorophenol in a previous paper (Oswald et al., 2005) and the aim of this paper is to complete our survey of the monofluoroand monochlorophenols.

The packing in the crystal structures of monoalcohols has been studied by Brock \& Duncan (1994) and Taylor \& Macrae (2001). Alcohols with bulky $R$ groups aggregate around three-, four- or sixfold screw, rotation or rotoinversion axes or crystallize in low-symmetry space groups with $Z^{\prime}>1$. Small alcohols tend to pack about $2_{1}$ axes or glide planes. Our previous work has shown that phenol, 2-chlorophenol and 4-fluorophenol behave like bulky alcohols at low temperature (packing about a pseudo-threefold axis in $P 2_{1}$, a $3_{2}$ axis in $P 3_{2}$ or a $\overline{3}$ site in $R \overline{3}$, respectively), but behave as small alcohols, packing about $2_{1}$ axes or glide planes, at high pressure.

3-Chlorophenol behaves typically, and packs with two independent molecules about a $2_{1}$ axis to emulate a fourfold screw axis, although there are significant departures from this ideal arrangement. This structure is obtained both at lowtemperature $(283 \mathrm{~K})$ and high pressure $(0.1 \mathrm{GPa})$. The geometric parameters characterizing the packing were slightly different. All or most of the interactions would be expected to decrease in length on further application of pressure, but one disadvantage of the methods used here is that hydrostatic conditions are lost on crystallization under pressure (the liquid behaves as its own hydrostatic fluid). Increasing the pressure on such samples therefore tends to degrade them.

3-Fluorophenol is unique in this series of compounds in crystallizing at ambient pressure like a small alcohol and forming chains disposed about a $2_{1}$ screw axis. The $\mathrm{O} \cdots \mathrm{O}$ distances in the chain are slightly longer than in the other phenols studied here and this may reflect steric effects in neighbouring phenyl groups. The structure is stabilized by F..H interactions. The same phase is obtained on crystallization at $0.12 \mathrm{GPa}$.

Both 2-fluorophenol and 4-chlorophenol exhibit more interesting phase behaviour with increasing pressure. Neither adopts the packing motif associated with small alcohols at high pressure; nor do they form very regular helical structures at ambient pressure.
The structure of 2-fluorophenol phase $\mathrm{I}$ at $150 \mathrm{~K}$ is depicted in Fig. 6. The packing is characterized by chains built by $\mathrm{OH} \cdots \mathrm{OH} \cdots$ hydrogen bonds. Pairs of molecules are linked across inversion centres; each pair is bridged by molecules disordered about twofold axes. The pairs of molecules connected across the inversion centres have a similar spatial relationship to a pair of molecules related by a glide plane or $2_{1}$ axis, as in the structures of small alcohols. The steric effect of the 2-fluorine atom means that this relationship cannot be propagated further and the other molecules in the chain are rotated about the chain axis (the $c$ direction) by $c a 90^{\circ}$. The 2-fluorine atoms point towards the chain axis and there is no scope for the formation of stabilizing $\mathrm{F} \cdots \mathrm{H}$ contacts either within the chains or between them.

A different polymorph (phase II) was obtained for 2fluorophenol at $0.36 \mathrm{GPa}$. The crystal of this phase was stable only above $c a 363 \mathrm{~K}$ and data were obtained at $403 \mathrm{~K}$. The structure has two molecules in the asymmetric unit and is also characterized by $\mathrm{OH} \cdots \mathrm{OH} \cdots$ chain formation. In projection the chain resembles a fourfold helix, but the repeat along the chain is irregular. The most important difference between this phase and phase $\mathrm{I}$ is the presence of $\mathrm{F} \cdots \mathrm{H}$ interactions which occur both within the hydrogen-bonded chains and between them. Thalladi et al. (1998) have shown that the $\mathrm{C}-\mathrm{H} \cdots \mathrm{F}$ interactions are as important as $\mathrm{C}-\mathrm{H} \cdots \mathrm{O}$ interactions in structure stabilization. The observation that more of these weak interactions are formed at high pressure is consistent with results observed in other high-pressure studies, for example, on glycine, where high-pressure induces extensive $\mathrm{CH} \cdots \mathrm{O}$ hydrogen-bond formation (Dawson et al., 2005). A feature exhibited by the high-pressure structures of 2-chlorophenol and 4-fluorophenol is the lengthening of $\mathrm{OH} \cdots \mathrm{OH} \cdots$ hydrogen bonds in order to accommodate more efficient packing motifs. A similar feature is observed in phase II of 2-fluorophenol, in which one $\mathrm{O} \cdots \mathrm{O}$ interaction is 3.097 (3) A.

The two phases of 4-chlorophenol presented here have been described previously and these structures are redeterminations. Phase I, which is based on an irregular helical arrangement of molecules linked by $\mathrm{OH} \cdots \mathrm{OH}$ interactions, crystallizes at ambient pressure from the melt. Under a modest pressure of $0.02 \mathrm{GPa}$, crystallization from the melt yields phase II, which is based on cyclic hydrogen-bonded tetramers. In both phases the $\mathrm{Cl}$ atoms link the hydrogen-bonded units together through $\mathrm{C}-\mathrm{Cl} \cdots \mathrm{H}$ interactions. These are somewhat shorter in phase II than in phase I. Moreover, the $R_{4}^{4}(8)$ ring has inversion symmetry rather than the $\overline{4}$ symmetry that might be expected for such a motif for a bulky alcohol. Neighbouring phenyl groups approach each other with centroid-centroid distances of $6.22 \AA$, mediated by a $\mathrm{CH}$ - aryl hydrogen bond. The more extensive set of contacts in phase II and its more efficient packing of phenyl groups perhaps explains the preference for this phase at high pressure. 


\section{Conclusions}

The behaviour of the 3-chloro, 3-fluoro-, 4-chloro- and 2fluorophenol show a departure from the behaviour observed in phenol, 2-chlorophenol and 4-fluorophenol. The transition to a small alcohol packing from bulky alcohol packing is not seen in any of the former compounds. In general, we find that they crystallize in pseudo-helices and ring motifs, although these are markedly distorted from three- or fourfold symmetry often observed in alcohol structures. Only one compound, 2fluorophenol, shows a phase that is only stable at high pressure. Those compounds that do undergo a phase transition, 4chlorophenol and 2-fluorophenol, show a transition to a phase which possesses a greater number of weaker intermolecular contacts in the form of $\mathrm{CH} \cdots \pi$ and $\mathrm{C}-\mathrm{H} \cdots \mathrm{F}$ interactions, respectively.

We thank the EPSRC, the Cambridge Crystallographic Data Centre and the University of Edinburgh for funding.

\section{References}

Allan, D. R., Clark, S. J., Dawson, A., McGregor, P. A. \& Parsons, S. (2002). Acta Cryst. B58, 1018-1024.

Allen, F. H. (2002). Acta Cryst. B58, 380-388.

Allen, F. H. \& Motherwell, W. D. S. (2002). Acta Cryst. B58, 407-422.

Altomare, A., Cascarano, G., Giacovazzo, C. \& Guagliardi, A. (1993). J. Appl. Cryst. 26, 343-350.

Betteridge, P. W., Carruthers, J. R., Cooper, R. I., Prout, K. \& Watkin, D. J. (2003). J. Appl. Cryst. 36, 1487.

Blessing, R. H. (1995). Acta Cryst. A51, 33-38.

Blessing, R. H. (1997). J. Appl. Cryst. 30, 421-426.

Boese, R. \& Nussbaumer, M. (1994). In Correlations, Transformations, and Interactions in Organic Crystal Chemistry, edited by D. W. Jones \& A. Katrusiak, pp. 20-37. International Union of Crystallography, Crystallographic Symposia 7. Oxford University Press.

Brock, C. P. \& Duncan, L. L. (1994). Chem. Mater. 6, 1307-1312.

Bruker-Nonius (2003). SAINT, Version 7. Bruker-Nonius Inc., Madison, Wisconsin, USA.

Bruno, I. J., Cole, J. C., Edgington, P. R., Kessler, M., Macrae, C. F., McCabe, P., Pearson, J. \& Taylor, R. (2002). Acta Cryst. B58, 389397.

Cosier, J. \& Glazer, A. M. (1986). J. Appl. Cryst. 19, 105-107.
Crystal Impact (2004). DIAMOND, Version 3a. Crystal Impact, Postfach 1251, 53002 Bonn, Germany; http://www.crystalimpact.com/diamond.

David, W. I. F., Shankland, K., Cole, J., Maginn, S., Motherwell, W. D. S. \& Taylor, R. (2001). DASH User's Manual. Cambridge Crystallographic Data Centre, Cambridge, UK.

Dawson, A., Allan, D. R., Belmonte, S. A., Clark, S. J., David, W. I. F., McGregor, P. A., Parsons, S., Pulham, C. R. \& Sawyer, L. (2005). Accepted for publication.

Dawson, A., Allan, D. R., Parsons, S. \& Ruf, M. (2004). J. Appl. Cryst. 37, 410-416.

Farrugia, L. J. (1999). J. Appl. Cryst. 32, 837-838.

Larson, A. C. (1970). Crystallogr. Comput. Proc. Int. Summer Sch. pp. 291-294.

Malone, J. F., Murray, C. M., Charlton, M. H., Docherty, R. \& Lavery, A. J. (1997). J. Chem. Soc. Faraday Trans. 93, 3429-3436.

Merrill, L. \& Bassett, W. A. (1974). Rev. Sci. Instrum. 45, 290-294.

Mezouar, M. (2001). High Pressure Powder Diffraction Using Synchrotron Radiation. British Crystallographic Association Physical Crystallography Group Meeting, Darebury Laboratory, Warrington, UK. November 2001.

Oswald, I. D. H., Allan, D. R., Day, G. M, Motherwell, W. D. S. \& Parsons, S. (2005). Accepted for publication.

Parsons, S. (2004a) ECLIPSE. The University of Edinburgh, Edinburgh, UK.

Parsons, S. (2004b) SHADE. The University of Edinburgh, Edinburgh, UK.

Perrin, P. M. \& Michel, P. (1973a). Acta Cryst. B29, 253-258.

Perrin, P. M. \& Michel, P. (1973b). Acta Cryst. B29, 258-263.

Sands, D. E. (1995). Vectors and Tensors in Crystallography, ch. 2. New York: Dover.

Sheldrick, G. M. (1997). SHELXL97. University of Göttingen, Germany.

Sheldrick, G. M. (2001). SHELXTL, Version 6.01. University of Göttingen, Germany and Bruker-Nonius Inc., Madison, Wisconsin, USA.

Sheldrick, G. M. (2004). SADABS, Version 2004-1. University of Göttingen, Germany, Bruker-AXS, Madison, Wisconsin, USA.

Sparks, R. A. (2000). GEMINI, Version 1.01. Bruker-AXS, Madison, Wisconsin, USA.

Spek, A. L. (2002). PLATON. Utrecht University, The Netherlands. Taylor, R. \& Macrae, C. F. (2001). Acta Cryst. B57, 815-827.

Thalladi, V. R., Weiss, H.-C., Blaser, D., Boese, R., Nangia, A. \& Desiraju, G. R. (1998). J. Am. Chem. Soc. 120, 8702-8710.

Watkin, D. J., Pearce, L. \& Prout, C. K. (1993). CAMERON. Chemical Crystallography Laboratory, University of Oxford, England. 